\title{
Mecanismo de centralização: da insuficiência placentária à adaptação circulatória fetal
}

\author{
Brain sparing effect: from placental insufficiency to fetal circulatory \\ adaptation
}

Palavras-chave

Fluxometria por laser-dopple Insuficiência placentária Circulação placentária Circulação sanguínea

Feto

Keywords

Laser-doppler flowmetry Placental insufficiency Placental circulation Blood circulation Fetus

Correspondência:

Juliana Marques Simões Villas-Bôas Departamento de Ginecologia, Obstetrícia e Mastologia da UNESP Distrito de Rubião Júnior, $s / n$ CEP 18618-970 - Botucatu / SP E-mail: julivillasboas@hotmail.com

Recebido

$2 / 7 / 08$

\section{Resumo}

A aplicação e o desenvolvimento da doplervelocimetria obstétrica apresentam base para conhecimento da insuficiência placentária e comprovam o comportamento dinâmico da circulação fetal em regime de hipóxia. Na prática clínica, tornou-se quase rotineira a necessidade de se avaliar a hemodinâmica em três territórios vasculares envolvidos na gestação: artérias uterinas, umbilical e cerebral média. Em linhas gerais, a artéria cerebral expressa o balanço entre a oferta de oxigênio nas uterinas e a captação pelas umbilicais. Atualmente, quando este balanço é desfavorável, procura-se ainda conhecer a reserva cardíaca fetal pelo estudo do ducto venoso. Contudo, precisar e interpretar índices de resistência vascular nem sempre é tarefa fácil. $\bigcirc$ ponto de partida é ter em mente os fundamentos sobre os quais se assenta o papel da doplervelocimetria para a avaliação do bem-estar fetal.

\section{Abstract}

The application and development of obstetric Dopplervelocimetry provide a basis for the investigation of placental insufficiency and demonstrate the dynamic behavior of fetal circulation during hypoxia. In clinical practice, assessing hemodynamics in three vascular regions involved in pregnancy, namely the uterine, umbilical and middle cerebral arteries, has become routine. Roughly, the cerebral artery expresses the balance between uterine artery oxygen supply and umbilical artery oxygen uptake. Currently, when such balance is unfavorable, the fetal cardiac reserve is investigated by assessing the venous duct. However, determining and interpreting vascular resistance indexes is not an easy task. The starting point is to know the physiopathology of placental insufficiency and fetal circulatory adaptation through which Doppler confirmed its role in the assessment of fetal well-being.
'Professora Substituta do Departamento de Ginecologia e Obstetrícia da Faculdade de Medicina de Botucatu da Universidade Estadual Paulista "Júlio de Mesquita Filho" UNESP - Botucatu (SP), Brasil.

2Professora Assistente Doutora do Departamento de Ginecologia e Obstetrícia da Faculdade de Medicina de Botucatu da Universidade Estadual Paulista "Júlio de Mesquita Filho" UNESP - Botucatu (SP), Brasil.

3Professor Assistente Doutor; Responsável pelo Setor de Ultra-sonografia e Medicina Fetal da Faculdade de Medicina de Botucatu da Universidade Estadual Paulista "Júlio de Mesquita Filho" UNESP - Botucatu (SP), Brasil. 


\section{Introdução}

Desde sua aplicação na obstetrícia, a doplervelocimetria fomentou descobertas sobre a fisiologia e a fisiopatologia da circulação fetal. Uma delas, difundida nas últimas duas décadas e bastante transformadora da prática clínica, é o fenômeno de redistribuição de fluxo sangüíneo pelo qual o feto responde à hipóxia, denominado mecanismo de centralização. Em outras palavras, observou-se que condições inadequadas de oxigenação intra-uterina desencadeiam processos de adaptação circulatória, os quais permitem ao feto preservar tecidos e funções vitais, aumentando a longevidade da gestação.

O emprego da doplerfluxometria em obstetrícia foi relatado em 1977. Nesta publicação inicial, as características hemodinâmicas da circulação na artéria umbilical foram descritas aplicando-se o Doppler contínuo, instrumento semelhante ao sonar, utilizado na ausculta dos batimentos cardíacos fetais, aqui associado ao registro gráfico das ondas de velocidade de fluxo ${ }^{1}$. A localização mais precisa da estrutura vascular tornou-se possível com o Doppler pulsátil, onde a imagem da estrutura anatômica é observada junto ao traçado do fluxo sangüíneo ${ }^{2}$. Desde então, com a evolução dos recursos tecnológicos, acumulam-se relatos que associam o Doppler das circulações materna e fetal a complicações clínicas na gestação ${ }^{3-6}$.

A compreensão da hemodinâmica fetal remete ao conhecimento do processo de placentação e modificações circulatórias no organismo materno, com estabelecimento da circulação útero-placentária.

\section{Circulação útero-placentária}

Durante a gestação, trocas nutricionais e gasosas ocorrem na placenta, interface física entre mãe e feto. Para garantir o crescimento, o desenvolvimento e a sobrevivência intra-uterina, a placenta induz modificações circulatórias que acabam por direcionar ao útero maior volume de sangue. Estudos de anatomia patológica descreveram as unidades estruturais, sítio desta transformação ${ }^{7}$ : as arteríolas espiraladas, ramos terminais das artérias uterinas, as quais atuam como canais de comunicação com a cavidade do útero e o espaço interviloso. Representam, dessa forma, estruturas onde se dá a ligação sangüínea da mãe com o feto.

A boa adaptação materna à gestação ${ }^{8}$, do ponto de vista circulatório, compreende a "modificação fisiológica" no perfil hemodinâmico do útero, que passa a demonstrar baixa resistência ao fluxo sangüíneo, baixa reatividade vasomotora e alta complacência vascular, favorecendo a oferta de substratos para o feto ${ }^{7}$. Tal modificação depende de adequada interação entre miométrio e trofoblasto, este constituído de células placentárias que apresentam antígenos paternos. Assim sendo, esta interação parece depender de fatores imunológicos ${ }^{9}$, modulando a atividade migratória do citotrofoblasto em profundidade no leito placentário, rumo às arteríolas espiraladas ${ }^{7,10}$.

A adaptação vascular induzida pela placenta acontece em dois estágios, um no primeiro e outro no segundo trimestre de gestação, configurando a primeira e a segunda onda de invasão do trofoblasto.

O início da primeira onda ocorre na quinta semana de gestação ${ }^{11}$. Neste processo, as arteríolas espiraladas sofrem infiltração intersticial e endovascular das células trofoblásticas, com vasodilatação e progressiva substituição da camada íntima por material fibrinóide. Nesta etapa, as alterações estão limitadas ao endotélio do segmento intradecidual ${ }^{7,10,12}$.

$\mathrm{Na}$ segunda onda, entre a $16^{\mathrm{a}}$ e a $20^{\mathrm{a}}$ semana de gestação, a invasão progride para segmentos mais profundos, na intimidade do miométrio. A camada músculoelástica das arteríolas espiraladas é substituída por tecido fibrinóide e fibroso, transformando-se nas artérias úteroplacentárias ${ }^{7,10}$.

A doplerfluxometria das artérias uterinas apresenta, durante este processo, modificações progressivas caracterizadas por aumento da velocidade máxima e dos fluxos de volume, expressando a diminuição da resistência vascular. O resultado na onda de velocidade de fluxo deve ser observado após 24 a 26 semanas de gestação, pelo aumento da velocidade diastólica e desaparecimento da incisura aórtica. Tal achado indica bom estabelecimento da interface de troca materno-fetal e adequada adaptação circulatória à gestaçãos 5 .

A migração incompleta do trofoblasto explica a insuficiência placentária e a seqüência de eventos relacionados à má adaptação circulatória na gestação ${ }^{7,8}$. Neste modelo, os efeitos serão percebidos em intensidades diferentes: na mãe, pelas síndromes hipertensivas; no ambiente intrauterino, pelo oligoâmnio, aceleração de maturidade, infartos e descolamentos placentários, e, no feto, pela restrição de crescimento e fenômenos de redistribuição de fluxo sangüíneo, incluindo o mecanismo de centralização.

A insuficiência vascular placentária parece decorrer de inadequada interação entre trofoblasto e tecidos uterinos, provocando alterações qualitativas e quantitativas das arteríolas espiraladas do leito placentário ${ }^{10}$. As modificações fisiológicas ficam restritas ao segmento decidual, não sendo observadas na porção miometrial das arteríolas espiraladas. Portanto, não ocorre a segunda onda de invasão trofoblástica ${ }^{9,11}$.

Observa-se, ainda, nessa situação, redução no total de vasos que irrigam o espaço interviloso ${ }^{10}$, oclusão vascular por material fibrinóide e infiltrado perivascular mononuclear, em processo semelhante ao de aterose ${ }^{13}$. Como conseqüência ocorre isquemia, liberação de citotoxinas e outras alterações que consolidam a agressão vascular. Esse 

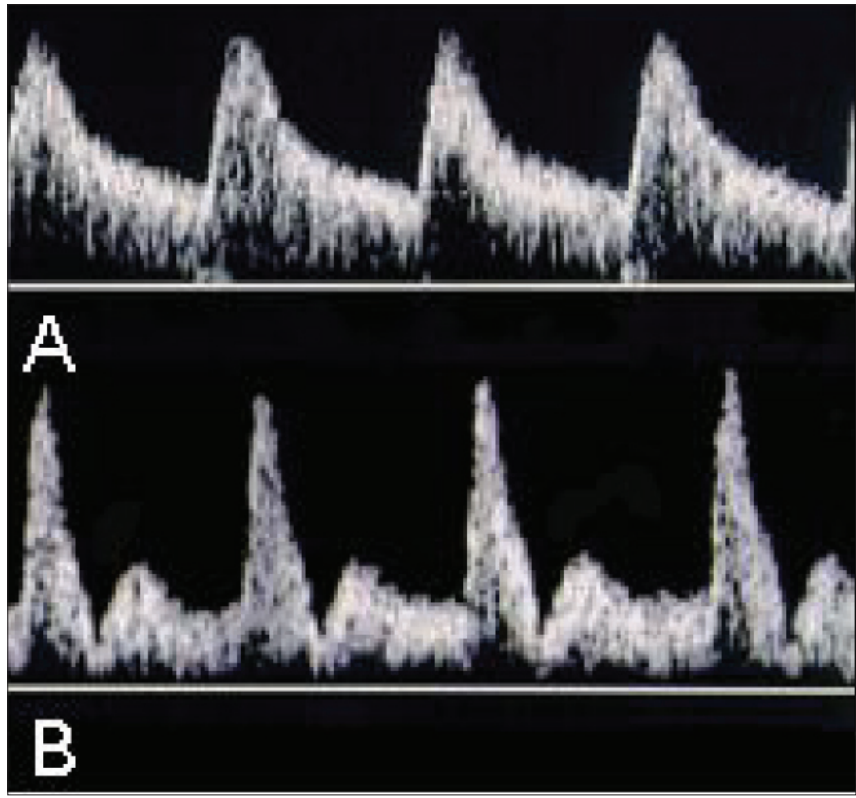

Figura 1 - Onda de velocidade de fluxo na artéria uterina. (A) normal (após segunda onda de invasão trofoblástica). (B) incisura protodiástólica

processo leva à redução do fluxo de troca materno-fetal no espaço interviloso e manutenção do perfil hemodinâmico presente antes da gestação, caracterizado por alta resistência ao fluxo sangüíneo e reatividade a estímulos vasoativos e baixa complacência vascular ${ }^{10}$.

$\mathrm{Na}$ doplervelocimetria das artérias uterinas, observa-se persistência da incisura aórtica e baixa velocidade diastólica, mantendo-se o padrão de resistência elevada após a $24^{\mathrm{a}}$ ou $26^{\mathrm{a}}$ semanas de gestação (Figura 1 ). Na literatura, está bem documentada a associação destas características doplervelocimétricas a complicações gestacionais como pré-eclâmpsia, restrição de crescimento intra-uterino e prematuridade $\mathrm{e}^{14-17}$.

\section{Circulação fetal e feto-placentária}

A distribuição do oxigênio no feto inicia-se a partir do espaço interviloso, local da placenta ocupado pelo sangue materno que banha as vilosidades terciárias. Estas últimas correspondem a unidades vasculares de troca onde circula o sangue fetal em ramos terminais das artérias umbilicais. Uma vez oxigenado, o sangue das vilosidades dirige-se ao feto pela veia umbilical. Por este vaso apresentar o maior teor de oxigênio da circulação fetal, o encaminhamento do retorno venoso ao ventrículo esquerdo é fator decisivo no suprimento deste elemento a outros tecidos vitais, especialmente o cérebro, além do próprio coração ${ }^{18}$. Neste trajeto, a mistura com o sangue não oxigenado, e conseqüente redução no teor de oxigênio, é minimizada graças à existência de dois atalhos ou desvios, que devem desaparecer após o nascimento: o ducto venoso e o forame oval.

Cerca da metade do fluxo da veia umbilical é desviado pelo ducto venoso (primeiro desvio), circuito direto entre veia umbilical e cava inferior, contornando a microcirculação hepática. Neste território, observam-se os mesmos níveis de $\mathrm{pH}, \mathrm{pO}_{2}, \mathrm{pCO}_{2}$ e saturação de oxigênio, daqueles obtidos na placenta ${ }^{18}$. O ducto venoso é estreito, com menos de $2 \mathrm{~mm}$ de comprimento e com um terço do calibre da veia umbilical, características que o tornam capaz de projetar, em alta velocidade para a veia cava, o sangue oxigenado que alcança o átrio direito de forma a seguir curso preferencial para o átrio esquerdo através do forame oval (segundo desvio). Neste trajeto, é minimizada a mistura com o fluxo lento e de baixa pressão de oxigênio da veia cava inferior que, chegando ao átrio, desagua no ventrículo direito. Do átrio esquerdo, o sangue rico em oxigênio chega ao ventrículo esquerdo, aorta ascendente e, assim, miocárdio e cérebro ${ }^{19}$.

O sangue desoxigenado que entra no ventrículo direito perfunde os pulmões, sendo que a maior parte atravessa o ducto arterial, alcançando a aorta descendente após o local de emergência das artérias carótidas e coronárias. As artérias umbilicais, ramos da ilíaca interna, rumam à placenta para o reabastecimento com oxigênio e nutrientes, constituindo a circulação feto-placentária.

A insuficiência vascular placentária, qualquer que seja sua etiologia, determina obliteração progressiva das arteríolas do sistema viloso terciário, desencadeando alteração do fluxo de reabastecimento fetal ${ }^{20}$.

Os parâmetros doplervelocimétricos da artéria umbilical refletem diretamente a resistência vascular na placenta. Neste vaso, as ondas de velocidade de fluxo em uma gravidez normal têm padrão característico: baixa resistência e alta velocidade de fluxo anterior, tanto na sístole quanto na diástole. No primeiro trimestre, o fluxo diastólico final está ausente e aumenta com o tempo de gestação devido à redução da resistência vascular. Esta mudança reflete o crescimento e amadurecimento da placenta, com maior número de vilosidades terciárias e dilatação de seus capilares. Na insuficiência placentária, a obstrução das arteríolas umbilicais, destruição do tecido placentário nas áreas de infarto e descolamentos na superfície de troca provocam aumento da resistência ao fluxo sangüíneo. A onda de velocidade de fluxo da artéria umbilical perde de forma progressiva o componente diastólico final, podendo tornar-se ausente ou reverso, na medida em que progride a disfunção placentária ${ }^{15,21}$ (Figura 2).

Esse aumento da resistência vascular feto-placentária promove respostas adaptativas cardíacas à custa do retorno venoso fetal objetivando a manutenção do débito cardíaco. O mecanismo principal neste sistema é o de Franklin-Starling, no qual a distensão das fibras musculares cardíacas promove o aumento da força contrátil e conseqüente elevação da fração de ejeção cardíaca, mantendo-se assim, o débito cardíaco fetal 


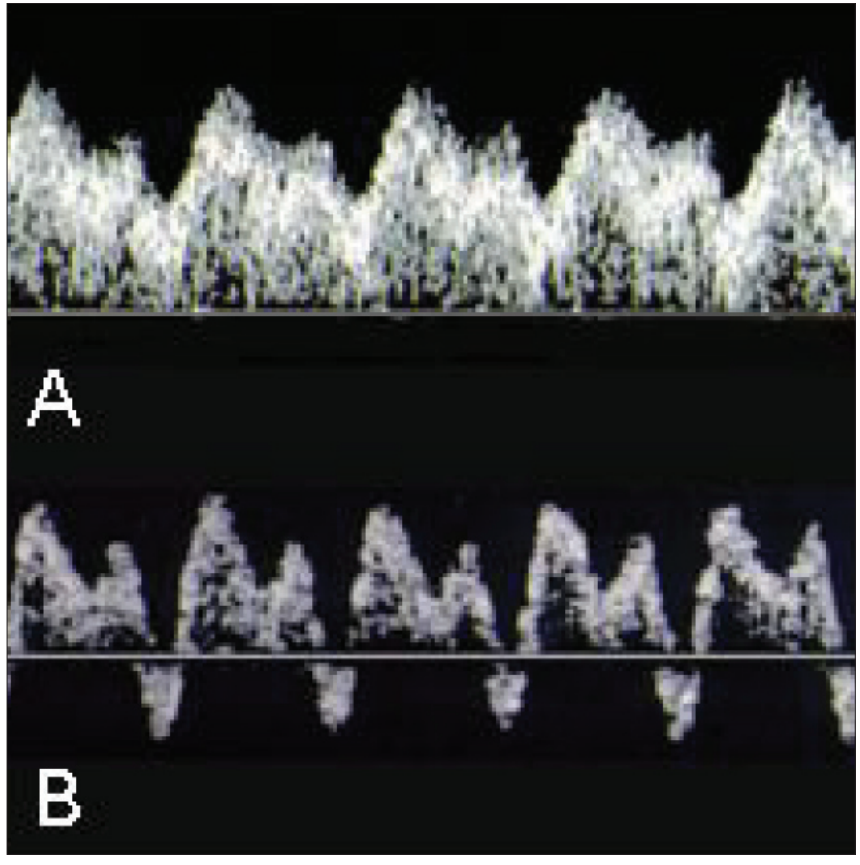

Figura 2 - Onda de velocidade de fluxo no ducto venoso. (A) normal; (B) onda " $a$ " invertida

às custas da pré-carga cardíaca. Ocorrendo distensão máxima da miofibrila cardíaca, a descompensação cardíaca é inevitável, frente ao incremento do retorno venoso, à crescente impedância vascular placentária e à oxigenação inadequada do miocárdio. O enchimento ventricular fica comprometido devido ao aumento do volume residual diástolico; o átrio direito distende-se, e provoca fluxo retrógrado, em direção a VCI, durante sua contração. Nesse momento, observa-se no ducto venoso, redução da velocidade na onda $A$, a qual, ao invés de expressar o fluxo anterógrado habitual da contração atrial, torna-se zero ou reversa na falência do miocárdio ${ }^{22-24}$ (Figura 3).

\section{Mecanismo de centralização}

O conceito da centralização e suas repercussões sobre o feto foram bem definidos por Saling no final da década de $1960^{25}$. A técnica Doppler permitiu investigação não invasiva, estabelecendo conhecimento mais aprofundado dos sinais circulatórios presentes na hipóxia intra-uterina em fetos humanos. Assim, em 1986, Wladmiroff, Tong e Stewart ${ }^{19}$, foram pioneiros em descrever esse fenômeno, denominado "brain sparing effect".

Observou-se que a circulação fetal responde de forma adaptativa à insuficiência placentária. A redução do fornecimento de oxigênio desencadeia mecanismos de compensação no feto que preservam tecidos nobres, cujas funções mantêm atividades vitais. Dentre esses mecanismos pode-se citar: poliglobulia, glicólise anaeróbia
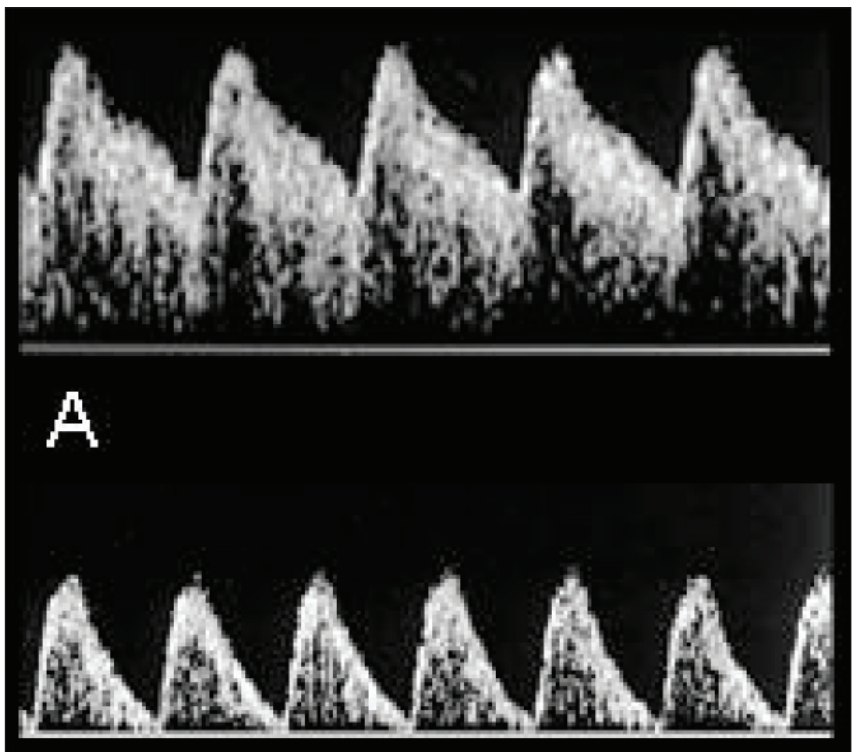

B
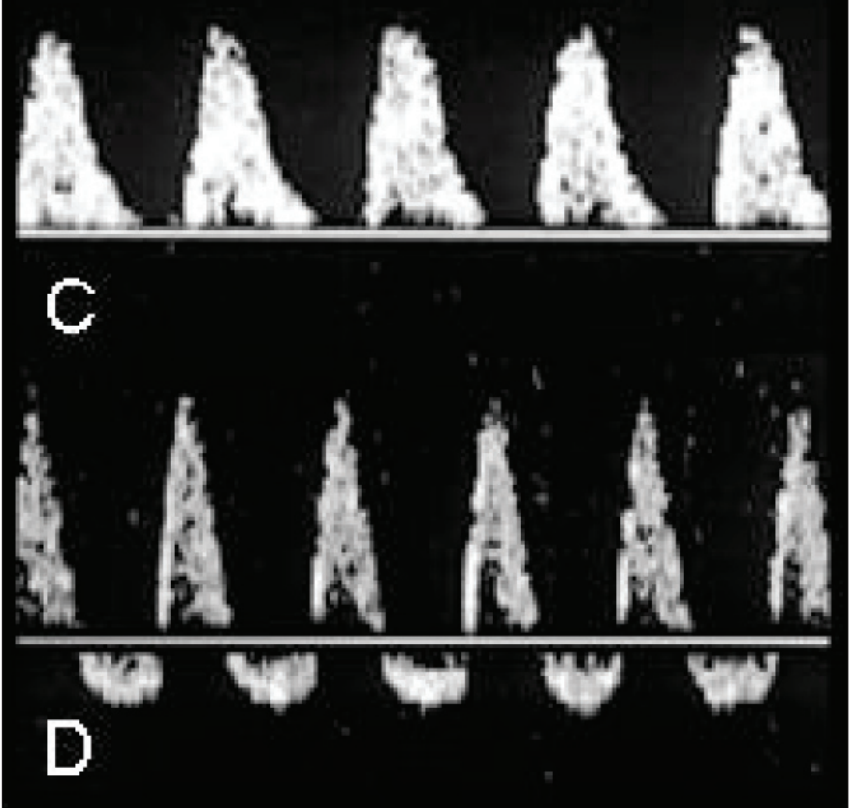

Figura 3 - Onda de velocidade de fluxo na artéria umbilical. (A) normal; (B) aumento de resistência - redução do componente diastólico; (C) diástole zero; (D) diástole reversa

e o mecanismo de centralização fetal ${ }^{23}$. A hipoxemia fetal também gera redistribuição do sangue no território venoso, aumentando em 30 a $65 \%$, a fração de sangue direcionada através do ducto venoso, contribuindo para manter a oxigenação dos órgãos nobres ${ }^{26}$.

O mecanismo de centralização é fenômeno de defesa ativo e temporário, desencadeado pelo estímulo de quimiorreceptores fetais na hipoxemia. Um maior fluxo de sangue é dirigido ao sistema nervoso central (centralização), miocárdio e glândulas adrenais pela redução da resistência vascular nestes territórios. Entretanto, outros órgãos sofrem prejuízo com a vasoconstrição 


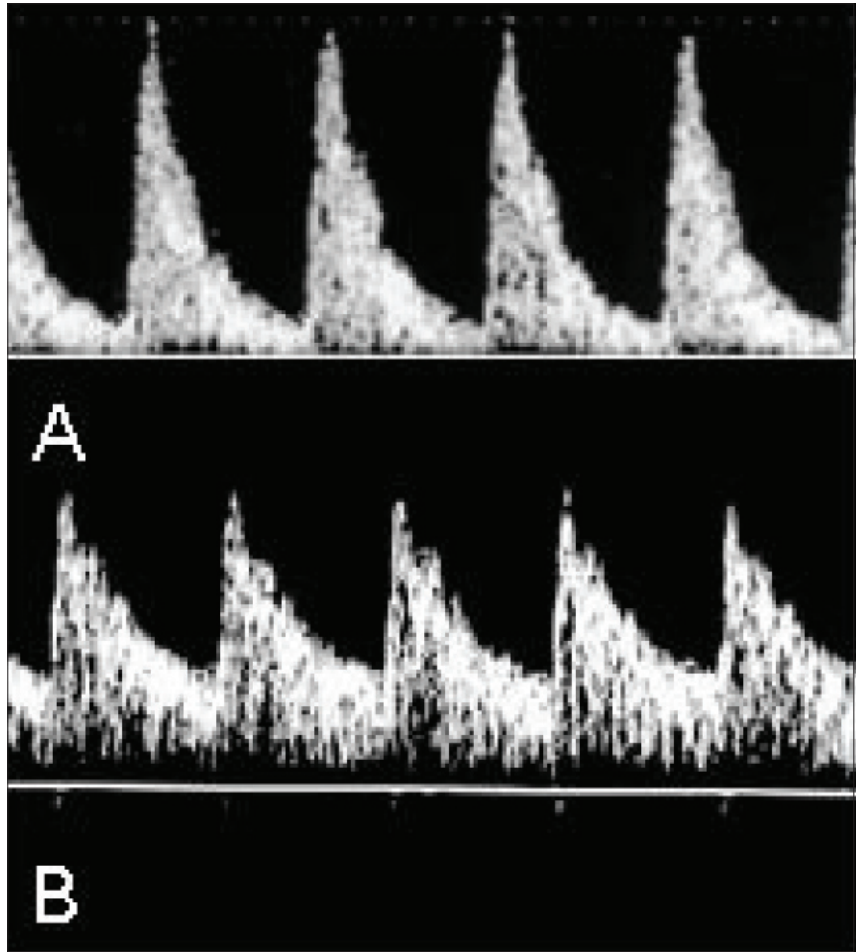

Figura 4 - Onda de velocidade de fluxo na artéria cerebral média. (A) normal; (B) vasodilatação

desencadeada por aumento da atividade simpática (adrenalina e noradrenalina). Assim, baixo fluxo sangüíneo no intestino, pulmões e rins resulta em enterocolite necrotizante, broncodisplasia pulmonar e insuficiência renal com oligoâmnio ${ }^{27-31}$.

A vasodilatação cerebral produz aumento de fluxo sangüíneo suficiente para que a oferta de oxigênio mantenha-se constante, preservando as atividades biofísicas do feto. Este fenômeno evidencia-se com a doplerfluxometria da artéria cerebral média pelo aumento da velocidade diastólica e redução dos índices de resistência ${ }^{19}$, com valores diretamente relacionados à gravidade da hipóxia ${ }^{29,32}$ (Figura 4).

O mecanismo de centralização torna-se insuficiente à medida que piora a hipoxemia, ocorrendo redução compensatória no consumo de oxigênio pelo tecido cerebral ${ }^{5}$.
A persistência desta condição desencadeia fenômeno de vasoplegia generalizada, caracterizado por modificações hemodinâmicas irreversíveis. $\mathrm{O}$ aparecimento de edema cerebral e aumento resultante na pressão intracraniana dificultam a perfusão sangüínea no cérebro. O edema cerebral, devido a acúmulo local de ácido lático, altera a permeabilidade da membrana celular, aumenta a pressão osmótica intracelular e conduz ao edema e necrose tissulares.

$\mathrm{Na}$ artéria cerebral média observa-se redução máxima da resistência vascular quando a $\mathrm{pO}_{2}$ do sangue da veia umbilical está entre dois e quatro desvios padrão abaixo da média normal para idade gestacional. À medida que se agrava o déficit de oxigênio, a resistência vascular tende a aumentar como resultado do edema cerebral, traduzindo vasoconstrição. Este fenômeno é conhecido como descentralização, sendo diagnosticado raramente, pouco antes do óbito intra-uterino ${ }^{29}$.

A centralização fetal traduz resposta compensatória do concepto. Entretanto, a duração e a eficácia desse mecanismo dependem da capacidade de adaptação e manutenção do equilíbrio hemodinâmico ${ }^{33}$. O tempo entre a instalação deste processo até o óbito é desconhecido, permanecendo entre um extremo e outro as complicações da morbidade perinatal relacionadas à asfixia ${ }^{34-35}$.

\section{Conclusão}

A insuficiência placentária é uma condição progressiva e irreversível, sendo tratada somente com a resolução da gestação. Porém, o momento ideal para se definir esta conduta é controverso e motivo de investigações. Envolve-se danos causados pela permanência intra-útero sob regime de hipóxia e complicações decorrentes da prematuridade. Neste sentido, a insuficiência placentária e a prematuridade caminham em sentidos opostos no estabelecimento do prognóstico fetal. Nestas situações, embora os avanços tecnológicos nas Unidades de Terapia Intensiva Neonatais tenham reduzido os índices de mortalidade e morbidade, decidir sobre a resolução da gestação permanece como um grande desafio.

\section{Referências}

1. FitzGerald DE, Drumm JE. Non-invasive measurement of human fetal circulation using ultrasound: a new method. $\mathrm{Br}$ Med J. 1977;2(6100): 1450-1

2. Gill RW. Quantitative flow measurement in deeplying vesseis using pulsed Doppler with the Octoson. In: White DN, Lyons EA, editors. Ultrasound in medicine. New York: Plenum Press; 1978. p. 341-8.
3. Baschat AA, Cosmi E, Bilardo CM, Wolf H, Berg C, Rigano S, et al. Predictors of neonatal outcome in early-onset placental dysfunction. Obstet Gynecol. 2007; $109(2$ Pt 1):253-61.

4. Francisco RP, Miyadahira S, Zugaib M. Predicting pH at birth in absent or reversed end-diastolic velocity in the umbilical arteries. Obstet Gynecol. 2006;107(5): 1042-8. 
5. Nicolaides KH, Rizzo G, Hecher K. Doppler studies in fetal hypoxemic hypoxia. In: Nicolaides KH, Rizzo G, Hecher K, editors. Placental and fetal Doppler. London: Parthenon; 2000. p. 67-88.

6. Sá RAM, Netto HC, Lopes LM, Barreto MV, Cabral ACV. Dopplerfluxometria do ducto venoso: relação com a gasometria em fetos prematuros com centralização de fluxo sangǘneo. Rev Bras Ginecol Obstet. 2003;25(4):261-8.

7. Brosens I, Robertson WB, Dixon HG. The physiological response of the vessels of the placental bed to normal pregnancy. J Pathol Bacteriol. 1967;93(2):569-79.

8. Wallenburg $\mathrm{HC}$. Prevention of hypertensive disorders in pregnancy. Clin Exp Hypertens Pregnancy. 1988;B7:121-37.

9. Robertson WB, Brosens I, Dixon HG. The pathological response of the vessels of the placental bed to hypertensive pregnancy. J Pathol Bacteriol. 1967;93(2):581-92.

10. Khong TY, De Wolf F, Robertson WB, Brosens I. Inadequate maternal vascular response to placentation in pregnancies complicated by pre-eclampsia and by small-for-gestational age infants. Br J Obstet Gynaecol. 1986;93(10):1049-59.

11. Valentin L, Sladkevicius P, Laurini R, Söderberg H, Marsal K. Uteroplacental and luteal circulation in normal first-trimester pregnancies: Doppler ultrasonographic and morphologic study. Am J Obstet Gynecol. 1996; 174(2):768-75.

12. Pijnenborg R, Bland JM, Robertson WB, Brosens I. Uteroplacental arterial changes related to interstitial trophoblast migration in early human pregnancy. Placenta. 1983;4(4):397-413.

13. Khong TY. Acute atherosis in pregnancies complicated by hypertension, small-for-gestational-age infants, and diabetes mellitus. Arch Pathol Lab Med. 1991; 115(7):722-5.

14. Trudinger BJ, Giles WB, Cook CM, Bombardieri J, Collins L. Fetal umbilical artery flow velocity waveforms and placental resistance: clinical significance. Br J Obstet Gynaecol. 1985;92(1):23-30.

15. Trudinger BJ, Giles WB, Cook CM. Uteroplacental blood flow velocity-time waveforms in normal and complicated pregnancy. Br J Obstet Gynaecol. 1985;92(1):39-45.

16. Campbell S, Pearce JM, Hackett G, Cohen-Overbeek T, Hernandez C. Qualitative assessment of uteroplacental blood flow: early screening test for high-risk pregnancies. Obstet Gynecol. 1986;68(5):649-53.

17. Papageorghiou AT, Yu CK, Cicero S, Bower S, Nicolaides KH. Secondtrimester uterine artery Doppler screening in unselected populations: a review. J Matern Fetal Neonatal Med. 2002;12(2):78-88.

18. Montenegro CAB, Rezende Filho J, Silva LGP. Centralização fetal. Femina. 1994;22(3):203-16,203-4,206-7,210-2, passim.

19. Wladmiroff JW, Tonge HM, Stewart PA. Doppler ultrasound assessment of cerebral blood flow in the human fetus. $\mathrm{Br} J$ Obstet Gynaecol. 1986;93(5):471-5.

20. Lenza GF, Silva LGP, Rezende Filho J, Castro KB, Montenegro CAB. Centralização fetal à luz da gasometria. J Bras Ginecol. 1997; 107(11/12):391-408.
21. Trudinger BJ, Stevens D, Connelly A, Hales JR, Alexander G Bradley $L$, et al. Umbilical artery flow velocity waveforms and placental resistance: the effects of embolization of the umbilical circulation. Am J Obstet Gynecol. 1987;157(6): 1443-8.

22. Coelho F, Aranda O, Belfort P, Rezende J. Dopplerfluxometria venosa fetal: considerações fisiopatológicas. Femina. 2003;31(1):9-15.

23. Melo VH, Cabral ACV, Netto HC. Detecção da centralização do fluxo sangüíneo fetal através do Doppler das artérias umbilical e cerebral média. J Bras Ginecol. 1993;103(1/2):27-34.

24. Carvalho FHC, Moron AF, Mattar R, Murta CGV, Santana RM, Torloni MR, et al. Índices veno-arteriais para predição da acidemia fetal ao nascimento em gestações com insuficiência placentária. Rev Bras Ginecol Obstet. 2004;26(8):641-7.

25. Saling E. New research results on the blood circulation of the newborn infant immediately fter birth.]Arch Gynakol. 1960;194:287. 306. German.

26. Vasconcelos RP, Aragão JRBF, Carvalho FHC, Feitosa FEL, Alencar Júnior CA. Resultados perinatais em fetos com alterações ao estudo dopplervelocimétrico. Femina. 2004;32(5):365-70.

27. Miyamura T, Masuzaki H, Miyamoto M, Ishimaru T. Comparison between the single deepest pocket and amniotic fluid index in predicting fetal distress in small-for-gestational age fetuses. Acta Obstet Gynecol Scand. 1997;76(2):123-7.

28. Bhatt AB, Tank PD, Barmade KB, Damania KR. Abnormal Doppler flow velocimetry in the growth restricted foetus as a predictor for necrotising enterocolitis. J Postgrad Med. 2002;48(3):1825; discussion 185.

29. Bilardo CM, Wolf H, Stigter RH, Ville Y, Baez E, Visser GH, et al. Relationship between monitoring parameters and perinatal outcome in severe, early intrauterine growth restriction. Ultrasound Obstet Gynecol. 2004;23(2): 119-25.

30. Franzin CMMO, Silva JLP, Marussi EF, Parmigiana SV. Centralização do fluxo sangǘneo fetal diagnosticado pela dopplervelocimetria em cores: resultados perinatais. Rev Bras Ginecol Obstet. $2001 ; 23(10): 659-65$

31. Nomura RMY, Francisco RPV, Miyadahira S, Zugaib M. Resultados perinatais em gestações com perfil biofísico fetal alterado. Rev Ginecol Obstet. 2001;12(2):74-8.

32. Vyas S, Nicolaides KH, Bower S, Campbell S. Middle cerebral artery flow velocity waveforms in fetal hypoxaemia. Br J Obstet Gynaecol. 1990;97(9):797-803.

33. Arduini $D$, Rizzo $G$, Romanini $C$. Changes of pulsatility index from fetal vessels preceding the onset of late decelerations in growthretarded fetuses. Obstet Gynecol. 1992;79(4):605-10.

34. Carrera JM. Estúdio hemodinâmico del deterioro fetal en el crecimiento intrauterino retardado. In: Carrera JM. Crecimiento normal y patológico. Barcelona: Masson; 1997. p. 389-99.

35. Villas-Bôas JMS. Gasometria da veia umbilical em fetos centralizados [dissertação]. Botucatu: Universidade Estadual Paulista "Júlio de Mesquita Filho"; 2007. 